\title{
Effects of the COVID-19 lockdown on glycaemic control in subjects with type 2 diabetes: the glycalock study
}

\author{
Luca D'Onofrio MD ${ }^{1}$ ( ) | Silvia Pieralice MD $^{2}$ | Ernesto Maddaloni MD ${ }^{1}$ | \\ Carmen Mignogna MD ${ }^{1}$ | Sara Sterpetti MD ${ }^{1}$ | Lucia Coraggio MD $^{1}$ | \\ Cecilia Luordi MD ${ }^{1}$ | Gloria Guarisco MD $^{3}$ | Gaetano Leto $\mathrm{MD}^{3}$ | \\ Frida Leonetti MD ${ }^{3}$ | Silvia Manfrini MD² | Raffaella Buzzetti MD ${ }^{1}$ ()
}

\footnotetext{
${ }^{1}$ Experimental Medicine Department, Sapienza University of Rome, Latina, Italy

${ }^{2}$ Endocrinology and Diabetes Unit, Campus Bio-Medico University of Rome, Rome, Italy

${ }^{3}$ Diabetes Unit, Department of MedicalSurgical Sciences and Biotechnologies, Santa Maria Goretti Hospital, Sapienza University of Rome, Latina, Italy

\section{Correspondence}

Luca D'Onofrio, MD, Experimental Medicine Department, Sapienza University of Rome, Viale Regina Elena 321, 00161 Rome, Italy.

Email: luca.donofrio@uniroma1.it

Funding information

No funding supported this study.
}

\begin{abstract}
Aim: To assess the effect of the coronavirus disease 2019 (COVID-19) lockdown on glycaemic control in subjects with type 2 diabetes (T2D).

Materials and Methods: In this observational, multicentre, retrospective study conducted in the Lazio region, Italy, we compared the differences in the HbA1c levels of 141 subjects with T2D exposed to lockdown with 123 matched controls with T2D who attended the study centres 1 year before. Basal data were collected from 9 December to 9 March and follow-up data from 3 June to 10 July in 2020 for the lockdown group, and during the same timeframes in 2019 for the control groups. Changes in $\mathrm{HbA} 1 \mathrm{c}(\triangle \mathrm{HbA} 1 \mathrm{c})$ and body mass index $(\triangle \mathrm{BMI})$ during lockdown were compared among patients with different psychological wellbeing, as evaluated by tertiles of the Psychological General Well-Being Index (PGWBS).

Results: No difference in $\triangle \mathrm{HbA} 1 \mathrm{c}$ was found between the lockdown and control groups (lockdown group $-0.1 \%[-0.5 \%-0.3 \%]$ vs. control group $-0.1 \%$ [ $-0.4 \%$ $-0.2 \%] ; p=.482)$. Also, no difference was found in $\triangle \mathrm{BMI}(p=.316)$ or $\Delta \mathrm{Glucose}$ $(p=.538)$. In the lockdown group, subjects with worse PGWBS showed a worsening of HbA1c ( $p=.041$ for the trend among PGWBS tertiles) and $\operatorname{BMI}(p=.022)$.

Conclusions: The COVID-19 lockdown did not significantly impact glycaemic control in people with T2D. People with poor psychological well-being may experience a worsening a glycaemic control because of restrictions resulting from lockdown. These findings may aid healthcare providers in diabetes management once the second wave of COVID-19 has ended.
\end{abstract}

\section{KEYWORDS}

glycaemic control, type 2 diabetes, psychological health, covid-19 lockdown 


\section{1 | INTRODUCTION}

The outbreak of coronavirus disease 2019 (COVID-19) has forced our society to face both the direct and indirect effects of the pandemic. The most important direct effect was a great emergency in healthcare management. The indirect effects of the pandemic are mainly caused by the lockdown and social distancing measures having a huge impact on economics and social life. ${ }^{1}$ An assessment of these effects on insulin-treated diabetes is paramount. While a detrimental effect on glycaemic control could be hypothesized, lockdown measures surprisingly played a null or even positive role in this group of patients, as highlighted in several observational studies from different countries. ${ }^{2,3}$ On the other hand, few and contrasting data were collected from patients affected by type 2 diabetes (T2D), showing both a detrimental effect of lockdown measures on glycaemic control ${ }^{4,5}$ and a positive effect. ${ }^{6}$ However, different study designs and inclusion criteria, a short observational period, single-centre observation and lack of appropriate controls could explain the heterogeneity of results.

For this reason, the primary aim of this study was to assess the effect of lockdown on glycaemic control in patients with T2D in a multicentre study enrolling matched controls. As a secondary aim, we explored whether psychological health, employment status, education, type of antidiabetes treatment and self-reported adherence to physical activity and dietary recommendations had an impact on diabetes management during lockdown.

\section{2 | MATERIALS AND METHODS}

\section{1 | Study design}

This was an observational retrospective study conducted in three diabetes centres in the Lazio region: the Diabetes Unit at Umberto Policlinico General Hospital, Sapienza University in Rome, the Endocrinology and Diabetes Unit at Campus Bio-Medico University Hospital in Rome and the Diabetes Unit at Santa Maria Goretti Hospital, Sapienza University, Polo Pontino in Latina. Consecutive subjects with T2D attending one of the three diabetes centres both within 3 months before lockdown (i.e. the basal visit occurred from 9 December 2019 to 9 March 2020) and within 1 month after lockdown (i.e. the followup visit occurred during 3 June-10 July 2020) were enrolled in the lockdown group. The exclusion criteria were any change in antidiabetes treatment during either the basal or telemedicine visit, severe acute respiratory syndrome coronavirus 2 (Sars-Cov-2) infection, type 1 diabetes, gestational diabetes, secondary diabetes (i.e. diabetes because of glucocorticoids, pancreatectomy, acromegaly, Cushing disease), new diabetes diagnosis and first referral to the centre for diabetes management. Accordingly, 141 subjects were enrolled in the lockdown group.

Age-, sex- and basal HbA1c-matched controls ( $n=123$ ) were selected among people with T2D attending any of the three study centres both during 9 December 2018-9 March 2019 (basal visit) and 3 June-10 July 2019 (follow-up visit), and were enrolled in the control group according to the same exclusion criteria used for the lockdown group.

\section{2 | Data collection}

Routine clinical and biochemical data collected during the diabetes visits and used in this study were age, sex, drug therapy, HbA1c, fasting blood glucose, body mass index (BMI), total cholesterol, HDL cholesterol and triglycerides. LDL cholesterol concentrations were estimated with the Friedewald formula: total cholesterol - [HDL + (triglycerides/5)]. Self-reported data about daily physical activity and adherence to the prescribed diet were also collected. Employment status during lockdown was categorized as follows: people who were retired; people who continued their normal work; people who continued their work from home (smart working); and, finally, people who were either unemployed or were housewives/househusbands.

Finally, to assess self-perceived psychological health and wellbeing during lockdown, we used the short version of the Psychological General Well-Being Index (PGWBS), validated for the Italian population. $^{7}$ The short version of the PGWBS is based on only six questions that explain $90 \%$ of the variance of the original questionnaire. ${ }^{7}$ The six questions evaluated different psychological domains of anxiety, vitality, depression, self-control and positive well-being; ${ }^{7}$ a higher score represents better psychological health.

\section{3 | Statistical analysis}

Descriptive statistics are presented for categorical variables as numbers with proportions, and for continuous variables as appropriate measures of central tendency and dispersion. Distributions of variables were tested for normality with the Shapiro-Wilk test. ANOVA and Kruskall-Wallis tests were used to analyse differences between groups for parametric and non-parametric continuous variables, respectively. Differences between basal and follow-up $(\Delta)$ between the lockdown and control groups, namely, $\Delta \mathrm{HbA} 1 \mathrm{c}, \Delta \mathrm{BMI}, \Delta \mathrm{Glucose}$, $\Delta$ Total cholesterol, $\Delta \mathrm{LDL}, \Delta \mathrm{HDL}$ and $\Delta$ Triglycerides, were tested using the $t$-test for continuous variables with parametric distribution, while the Mann-Whitney test was used for non-parametric variables. Categorical variables were compared with a $\chi^{2}$ or Fisher's exact test as appropriate.

For the primary aim of the study, we evaluated differences in $\triangle \mathrm{HbA} 1 \mathrm{c}$ between the groups. Differences in follow-up HbA1c were also reported for descriptive purposes. Differences in $\Delta$ between the groups in all other collected variables were also tested as exploratory analyses. If a statistical difference was observed, differences in the follow-up of that specific variable were also tested.

For subgroup analyses, tertiles of PGWBS, categories of employment status (as specified above), educational level (based on the presence or absence of any degree), type of antidiabetes treatment (treatment with or without multiple daily doses of insulin [MDI]) and adherence to the prescribed diet and to physical activity, were used. 
Based on a clinically relevant change in $\mathrm{HbA} 1 \mathrm{c}$ of $0.5 \%$ between the lockdown and control groups, we estimated that 123 patients were needed to detect a medium effect size (0.5) in the change of HbA1c from baseline with $80 \%$ power. IBM SPSS Statistics v. 21 software was used for data analysis and Prism 7.0 software was used for graphical representations.

\section{$2.4 \quad$ Ethics}

The study was performed in accordance with the Declaration of Helsinki, and the study procedures were approved by the institutions' ethics committees (ref. 5819/2020). The local ethics committees approved this retrospective observational study as minimal risk research using data collected for routine clinical practice and waived the requirement for informed consent, ensuring that the new privacy policy was followed.

\section{$3 \mid$ RESULTS}

\section{1 | Population features}

Of the 141 patients enrolled in the lockdown group, 61\% were male. The median (25th-75th percentile) age was 68.0 (61.0-74.0) years with a median disease duration of 13.0 (7.0-20.0) years. Among the 123 subjects enrolled in the control group, 61\% were male, with a median age of $69.0(63.0-76.0)$ years and a median disease duration of 13.0 (7.0-25.0) years. Except for basal BMI, which was slightly higher in the lockdown group, clinical characteristics did not differ between the two study groups (Table 1). Further, in the lockdown group, $54 \%$ of subjects were retired and $21 \%$ were unemployed. Among the workers, $8 \%$ continued their usual job during the lockdown period, whereas $10 \%$ of participants worked from home (smart working). Finally, the proportions of patients who reported continuing a regular physical activity and following a prescribed diet during the lockdown were $41 \%$ and $43 \%$, respectively.

\section{2 | Effects of lockdown on HbA1c and other variables}

No significant difference in terms of $\triangle \mathrm{HbA} 1 \mathrm{c}$ was found between the lockdown group and the control group (lockdown group $-0.1 \%$ [-0.5\%-0.3\%] vs. control group $-0.1 \%[-0.4 \%-0.2 \%] ; p=.482)$, as shown in Table 2. Accordingly, no absolute difference in follow-up HbA1c was found (lockdown group 7.3\% [6.6\%-8.0\%] vs. control group $7.4 \%$ [6.8\%-7.9\%]; $p=.482$ ).

As for the secondary aim of the study, no differences were found in $\triangle \mathrm{BMI}(p=.316), \Delta$ Glucose $(p=.538), \Delta \mathrm{HDL}(p=.142)$ or $\Delta$ Triglycerides $(p=.887)$ between the lockdown and control groups (Table 2). A significant difference was found in terms of $\Delta$ Total cholesterol (lockdown group -1 [-17-13] $\mathrm{mg} / \mathrm{dL}$ vs. control group -10 [-24-4] mg/dL; $p=.006$ ) and $\Delta \mathrm{LDL}$ (lockdown group -1 [-13$10] \mathrm{mg} / \mathrm{dL}$ vs. control group $-11[-30-2] \mathrm{mg} / \mathrm{dL} ; p<.001)$. However, no absolute differences were found in follow-up total cholesterol (lockdown group 165 [138-195] mg/dL vs. control group 171 [134199] $\mathrm{mg} / \mathrm{dL} ; p=.612$ ) or LDL (lockdown group 93 [66-118] mg/dL vs. control group $88[56-113] \mathrm{mg} / \mathrm{dL} ; p=.222$ ).

\section{3 | Psychological health and diabetes management during lockdown}

A significant difference between tertiles of PGWBS score was found for both $\triangle \mathrm{HbA} 1 \mathrm{c}(p=.041)$ and $\triangle \mathrm{BMI}(p=.022)$. In particular, subjects in the lowest tertile of the PGWBS score, namely, those with the poorest psychological well-being, showed a worsening in their metabolic control- $\triangle \mathrm{HbA} 1 \mathrm{c} \quad(0.2 \% \quad[-0.3 \%-0.6 \%]$ vs. $-0.2 \%[-0.9 \%-0.1 \%] ; p=.014)$ and $\Delta \mathrm{BMI}(0.0[-0.4-0.9]$ $\mathrm{kg} / \mathrm{m}^{2}$ vs. 0.0 [-0.9-0.3] $\mathrm{kg} / \mathrm{m}^{2} ; p=.043$ )-compared with patients in the highest tertile (i.e. those with better psychological wellbeing; Figure 1). Further, subjects in the middle tertile of the PGWBS score showed an increase in $\triangle B M I\left(0.3[-0.3-1.2] \mathrm{kg} / \mathrm{m}^{2}\right.$ vs. $0.0[-0.9-0.3] \mathrm{kg} / \mathrm{m}^{2} ; p=.008$ ) compared with patients in the highest tertile (Figure 1).

\subsection{Other subgroup analyses in the lockdown group}

After subdividing subjects enrolled in the lockdown group by employment categories, only retired patients showed an improvement in HbA1c during the lockdown (prelockdown HbA1c 7.4\% [6.8\%-8.1\%] vs. postlockdown HbA1c 7.3\% [6.4\%-7.9\%]; $p=.006$ ), while no differences were observed among workers $(p=.146)$, subjects who were smart working ( $p=.462$ ), or people who were unemployed $(p=.517$ ). Also, no differences were found in prelockdown and postlockdown HbA1c levels after subdividing patients by education level (patients with a degree, $p=.729$; patients without a degree, $p=.154$ ), treatment with MDI (patients with MDI, $p=.684$; patients without MDI, $p=.071$ ), self-reported physical activity (patients who reported physical activity, $p=.182$; patients who did not report physical activity, $p=.930$ ) or self-reported prescribed diet (patients who reported a correct diet, $p=.317$; patients who did not report a correct diet, $p=.775)$ during lockdown (Table S1).

\section{4 | DISCUSSION}

Most of the studies published during 2020 were focused on the acute effects of COVID-19; few studies explored the indirect effect of the pandemic on specific populations, especially in subjects with T2D. This is one of the first studies investigating the effect of lockdown on glycaemic control in patients affected by T2D. In particular, data 
TABLE 1 Baseline features

\begin{tabular}{|c|c|c|c|}
\hline & Lockdown group $(n=141)$ & Control group $(n=123)$ & $p$ \\
\hline Male, (n) \% & $61.0 \%(86)$ & $61.0 \%(75)$ & .998 \\
\hline Age, years & $68.0(61.0-74.0)$ & $69.0(63.0-76.0)$ & .604 \\
\hline Basal BMI, kg/m2 & $28.2(25.7-31.5)$ & $26.2(23.9-29.6)$ & $<.001$ \\
\hline PGWBS score & $18(15-23)$ & N/A & \\
\hline Basal HbA1c, \% & $7.4(6.9-8.2)$ & $7.3(6.8-7.9)$ & .157 \\
\hline Basal serum blood glucose, $\mathrm{mg} / \mathrm{dL}$ & $141(110-161)$ & $133(103-152)$ & .069 \\
\hline Basal total cholesterol, $\mathrm{mg} / \mathrm{dL}$ & $170(145-198)$ & $177(148-203)$ & .131 \\
\hline Basal HDL, mg/dL & $46(40-54)$ & $47(39-55)$ & .825 \\
\hline Basal triglycerides, $\mathrm{mg} / \mathrm{dL}$ & $123(94-159)$ & $123(93-166)$ & .944 \\
\hline Primary school, \% (n) & $28(39)$ & N/A & \\
\hline Secondary school, \% (n) & $38(54)$ & N/A & \\
\hline University, \% (n) & $9(12)$ & N/A & \\
\hline $\mathrm{PhD}$, specialization, \% (n) & $2(3)$ & N/A & \\
\hline \multicolumn{4}{|l|}{ Employment } \\
\hline Missing, (n) \% & $7(10)$ & N/A & \\
\hline Retired, (n) \% & $54(76)$ & N/A & \\
\hline Worker, (n) \% & $8(12)$ & N/A & \\
\hline \multicolumn{4}{|l|}{ Therapy } \\
\hline Diet alone, (n) \% & $2.1(3)$ & $3.2(4)$ & .702 \\
\hline Metformin, (n) \% & 83.0 (117) & $69.9(86)$ & .012 \\
\hline Sulphonylureas, (n) \% & $2.8(4)$ & $11.4(14)$ & .006 \\
\hline Glitazones, (n) \% & $2.1(3)$ & $5.7(7)$ & .130 \\
\hline DPP4-i, (n) \% & $13.5(19)$ & $16.3(20)$ & .525 \\
\hline GLP1-RA, (n) \% & 27.7 (39) & $20.3(25)$ & .165 \\
\hline SGLT2-i, (n) \% & $21.3(30)$ & $16.3(20)$ & .299 \\
\hline Basal insulin, (n) \% & $47.5(67)$ & $45.5(56)$ & .747 \\
\hline MDI, (n) \% & $19.9(28)$ & $22.0(27)$ & .676 \\
\hline
\end{tabular}

Abbreviations: BMI, body mass index; DPP4-i, dipeptidyl peptidase-4 inhibitor; GLP1-RA, glucagon-like peptide-1 receptor agonist; MDI, multiple daily doses of insulin; PGWBS, Psychological General Well-Being Index; SGLT2-i, sodium-glucose co-transporter-2 inhibitor.

observed in people experiencing lockdown measures were compared with data obtained from age- and gender-matched subjects enrolled during the same time period 1 year before the lockdown.

No significant difference in $\triangle \mathrm{HbA} 1 \mathrm{c}$ was found comparing subjects with stable therapy exposed to lockdown measures compared with controls.
These observations follow the same trend of previous findings in patients with insulin-treated diabetes. ${ }^{2,3}$ Recently, similar results were also obtained among Indian people with $\mathrm{T} 2 \mathrm{D},{ }^{6}$ despite the possible differential impact of ethnicity and geography in diabetes management. ${ }^{8}$ Conversely, another study conducted in Italy observed a significant increase of $\mathrm{HbA} 1 \mathrm{c}$ in $26 \%$ of patients enrolled during the first 
TABLE 2 Differences in characteristics between the patient and control groups

\begin{tabular}{llll} 
& Patients $(\mathbf{n}=\mathbf{1 4 1})$ & Controls $(\mathbf{n}=\mathbf{1 2 3})$ & $p$ \\
\hline$\Delta \mathrm{HbA} 1 \mathrm{c}, \%$ & $-0.1(-0.5-0.3)$ & $-0.1(-0.4-0.2)$ & .482 \\
\hline $\mathrm{BMI}, \mathrm{kg} / \mathrm{m} 2$ & $0.0(-0.7-0.5)$ & $0.0(-0.7-0.3)$ & .316 \\
\hline$\Delta$ Glucose, $\mathrm{mg} / \mathrm{dL}$ & $-6[-26-16]$ & $0[-23-17]$ & .538 \\
\hline Total cholesterol, $\mathrm{mg} / \mathrm{dL}$ & $-1[-17-13]$ & $-10[-24-4]$ & .006 \\
\hline$\Delta \mathrm{LDL}, \mathrm{mg} / \mathrm{dL}$ & $-1[-13-10]$ & $-11[-30-2]$ & $<.001$ \\
\hline$\Delta \mathrm{HDL}, \mathrm{mg} / \mathrm{dL}$ & $-1[-5-3]$ & $-2[-6-1]$ & .142 \\
\hline$\Delta$ Triglycerides, $\mathrm{mg} / \mathrm{dL}$ & $0[-19-22]$ & $3[-24-19]$ & .887 \\
\hline
\end{tabular}

Abbreviation: BMI, body mass index.
FIGURE 1 Differences in (A) $\triangle \mathrm{HbA} 1 \mathrm{c}$ and $(\mathrm{B}) \triangle \mathrm{BMI}$ by tertiles of the Psychological General Well-Being Index (PGWBS) score
(A)

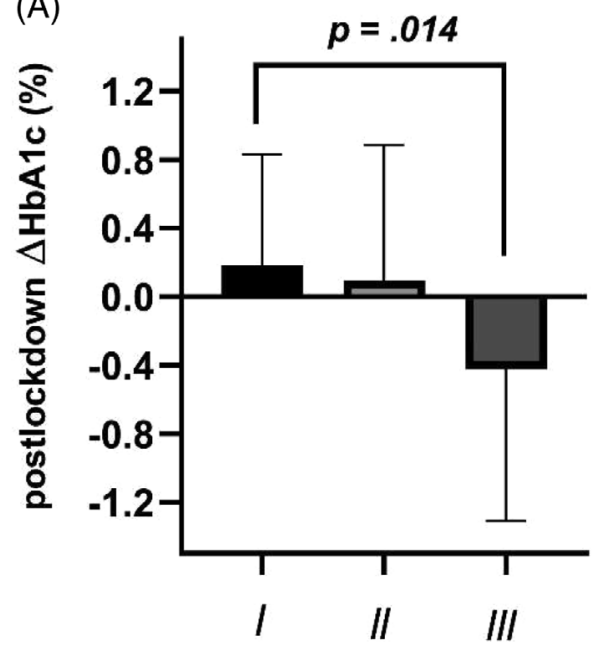

tertiles of PGWBS score

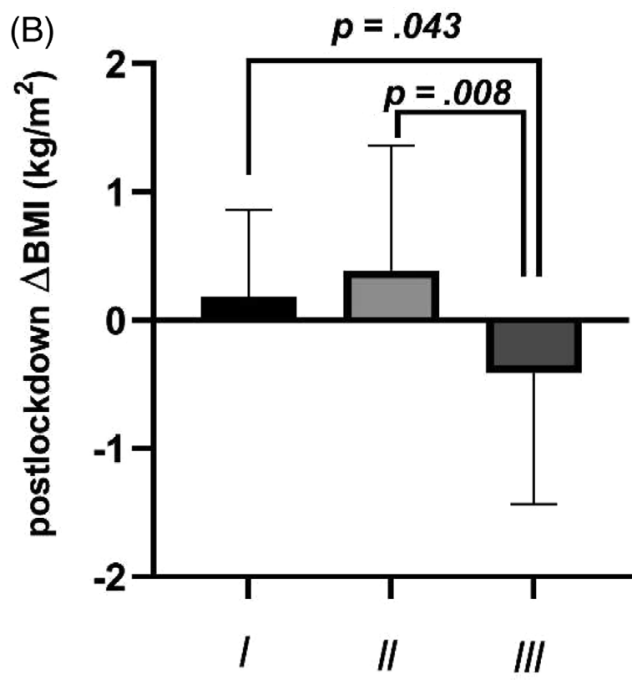

tertiles of PGWBS score
8 weeks of lockdown. ${ }^{4}$ A worsening of glycaemic control during the first phase of lockdown was also reported among Indian people by Khare and Jindal. ${ }^{9}$ Differences in timing, length and methods could potentially explain the different results across these studies. In addition, we can speculate that during the weeks of lockdown, a detrimental effect of restrictive measurements in diabetes management could be observed; however, after the first period of time, patients were able to cope with T2D and manage it as usual.

Overall, these results can be useful in clinical work planning, suggesting that patients with good prelockdown glycaemic control not requiring changes in antidiabetes therapy are able to cope with T2D, regardless of their education level and MDI treatment. Thus, during the time of physical distancing and rearrangement of resources, healthcare providers could reduce the effort in following up stable patients, for example, by extending follow-up appointments by a few months. The spared resources could then be used in the management of patients with T2D and relevant co-morbidities who are at an increased risk of developing a poor prognosis of COVID-19. ${ }^{10-12}$

Interestingly, among subjects exposed to lockdown measures, only retired patients showed an improvement in HbA1c. This finding is consistent with results observed in people with insulin-treated diabetes, ${ }^{3}$ especially patients with type 1 diabetes, ${ }^{13}$ suggesting that a more stable rhythm of life increases the time available to cope with daily management of diabetes. This speculation could be applicable not only to young people, but also to adults and old people.

We also noticed a higher percentage of metformin users and a lower percentage of sulphonylurea users among people in follow-up during the lockdown period. While this may be the result of the progressive reduction in sulphonylurea prescriptions in Italy, ${ }^{14}$ this observation might also highlight the relevance of using drugs with a low hypoglycaemic risk and with possible benefits for better COVID-19 outcomes ${ }^{15}$ during the lockdown.

As suggested by our subgroup analysis using a validated questionnaire (i.e. the PGWBS), an ability to cope with the pandemic is strictly related to the psychological stress experienced during lockdown. In this respect, Khare et al. reported that psychological stress because of the pandemic, as assessed by a self-designed questionnaire, was responsible for the worsening of glycaemic control. ${ }^{9}$ Psychological distress is commonly observed in countries facing the pandemic and adopting lockdown measures. ${ }^{16}$ The presence of chronic illness was one of the risk factors associated with mental distress. ${ }^{16}$ In particular, a report from South America observed that patients with diabetes 
showed a high prevalence of emotional distress as well as eating and sleeping disorders during lockdown. ${ }^{17}$ Further, data from India showed that subjects experiencing mental stress develop unhealthy dietary habits. ${ }^{18}$ Taken together, this evidence suggests that patients with diabetes are at an increased risk of mental issues related to the pandemic, which may then translate into a significant worsening of metabolic control, calling for healthcare systems to consider special psychological support for patients in this category.

Note that we found a significant difference in $\Delta$ Total cholesterol and $\Delta \mathrm{LDL}$ between the lockdown and control groups; however, the current study was not designed to evaluate these specific outcomes and we cannot exclude that some bias could have interfered with the results. Therefore, we should interpret these results with caution. First of all, we were not able to collect information about changing lipidlowering treatment. If so then we could speculate that the different results in $\Delta$ Total cholesterol and $\Delta L D L$ could be explained by the difficulty in contacting specialists, by patients exposed to lockdown measures, resulting in a reduction of prescriptions for lipid-lowering treatment.

The limitations of this study include the retrospective study design and self-reported data about adherence to prescribed diet and physical activity. Unfortunately, because of the nonpredictability of the pandemic, a prospective study design was not possible. The exclusion of patients who required intensification of antidiabetes therapy might have caused selection bias; however, this was necessary to isolate the effects of lockdown on glycaemic control, thus avoiding bias because of the introduction of new hypoglycaemic treatment. We also chose to stop study recruitment in mid-July to reduce any possible bias as a result of the distance in time from the end of the lockdown. Further, data on employment status, education and adherence to prescribed diet and physical activity were only available for a few patients in the control group, therefore not allowing sufficient controls for subgroup analyses. The study strengths include the multicentre design, thus reducing the bias of the single-centre study, and the availability of various data about key factors possibly influencing glycaemic control during lockdown. Further, another major strength was that data collected were compared with data from age- and gender-matched subjects enrolled during the same timeframe 1 year before, in order to obtain a control group that was not experiencing the effects of lockdown measures. We also collected information on telemedicine during the lockdown period, excluding possible bias resulting from an intervention by medical staff during the pandemic. Finally, we took advantage of this unique opportunity to study the effect of lockdown on glycaemic control as soon as the lockdown ended in Italy.

In conclusion, this study shows that there are no differences in diabetes control before and after lockdown in patients not requiring intensification of their usual hypoglycaemic treatment. Further, psychological stress could have a detrimental effect on glycaemic control. These results might be useful to help healthcare providers plan and organize diabetes management in the future once the second wave of COVID-19 has ended.

\section{ACKNOWLEDGEMENTS}

We thank Chiara Moretti and Antonio Siena for their help provided during the enrolment of patients. No funding supported this study.

\section{CONFLICT OF INTEREST}

All the authors declare no conflicts of interests related to this manuscript.

\section{AUTHOR CONTRIBUTIONS}

LDO: design, conducted data collection, analysis and manuscript writing. SP and CM: data collection and analysis. SS, LC, CL, GG and GL: data collection. EM: analysis and manuscript writing. $\mathrm{FL}$ and $\mathrm{SM}$ : revised the manuscript for critical content. RB: design, manuscript writing. All the authors gave approval for the final version of the work to be published.

\section{PEER REVIEW}

The peer review history for this article is available at https://publons. com/publon/10.1111/dom.14380.

\section{DATA AVAILABILITY STATEMENT}

Data available on request from the authors

\section{ORCID}

Luca D'Onofrio (D) https://orcid.org/0000-0003-3905-0139

Raffaella Buzzetti (D) https://orcid.org/0000-0003-1490-6041

\section{REFERENCES}

1. Hall G, Laddu DR, Phillips SA, Lavie CJ, Arena R. A tale of two pandemics: how will COVID-19 and global trends in physical inactivity and sedentary behavior affect one another? Prog Cardiovasc Dis. 2020;8:108-110

2. Capaldo B, Annuzzi G, Creanza A, et al. Blood glucose control during lockdown for COVID-19: CGM metrics in Italian adults with type 1 diabetes. Diabetes Care. 2020;43(8):e88-e89.

3. Maddaloni E, Coraggio L, Pieralice S, Carlone A, Pozzilli P, Buzzetti R. Effects of COVID-19 lockdown on glucose control: continuous glucose monitoring data from people with diabetes on intensive insulin therapy. Diabetes Care. 2020;43(8):e86-e87.

4. Biancalana E, Parolini F, Mengozzi A, Solini A. Short-term impact of COVID-19 lockdown on metabolic control of patients with wellcontrolled type 2 diabetes: a single-centre observational study. Acto Diabetol. 2020;21:1-6.

5. Önmez A, Gamsızkan Z, Özdemir Ş, et al. The effect of COVID-19 lockdown on glycemic control in patients with type 2 diabetes mellitus in Turkey. Diabetes Metab Syndr Clin Res Rev. 2020;14(6): 1963-1966.

6. Rastogi A, Hiteshi P, Bhansali A. Improved glycemic control amongst people with long-standing diabetes during COVID-19 lockdown: a prospective, observational, nested cohort study. Int J Diabetes Dev Ctries. 2020;40(4):476-481.

7. Grossi E, Groth N, Mosconi P, et al. Development and validation of the short version of the psychological general well-being index (PGWB-S). Health Qual Life Outcomes. 2006;4:88.

8. Maddaloni E, D'Onofrio L, Pozzilli P. Frailty and geography: should these two factors be added to the ABCDE contemporary guide to diabetes therapy? Diabetes Metab Res Rev. 2016;32(2): 169-175. 
9. Khare J, Jindal S. Observational study on effect of lock down due to COVID 19 on glycemic control in patients with diabetes: experience from Central India. Diabetes Metab Syndr. 2020;14(6):1571-1574.

10. Maddaloni E, D'Onofrio L, Alessandri F, et al. Cardiometabolic multimorbidity is associated with a worse Covid-19 prognosis than individual cardiometabolic risk factors: a multicentre retrospective study (CoViDiab II). Cardiovasc Diabetol. 2020;19(1):164.

11. Apicella M, Campopiano MC, Mantuano M, Mazoni L, Coppelli A, Del Prato S. COVID-19 in people with diabetes: understanding the reasons for worse outcomes. Lancet Diabetes Endocrinol. 2020;8: 782-792.

12. Maddaloni E, D'Onofrio L, Alessandri F, et al. Clinical features of patients with type 2 diabetes with and without Covid-19: a case control study (CoViDiab I). Diabetes Res Clin Pract. 2020;169: 108454.

13. Potier L, Hansel B, Larger E, et al. Stay-at-home orders during the COVID-19 pandemic, an opportunity to improve glucose control through behavioral changes in type 1 diabetes. Diabetes Care. 2020; 44:dc202019.

14. AMD. Annali AMD 2020. https://aemmedi.it/wp-content/uploads/ 2020/10/Annali-nuova-versione-2020_1-ok.pdf. Accessed March 30, 2021.

15. Hariyanto TI, Kurniawan A. Metformin use is associated with reduced mortality rate from coronavirus disease 2019 (COVID-19) infection. Obes Med. 2020;19:100290.
16. Xiong J, Lipsitz O, Nasri F, et al. Impact of COVID-19 pandemic on mental health in the general population: a systematic review. J Affect Disord. 2020;277:55-64.

17. Alessi J, de Oliveira GB, Franco DW, et al. Mental health in the era of COVID-19: prevalence of psychiatric disorders in a cohort of patients with type 1 and type 2 diabetes during the social distancing. Diabetol Metab Syndr. 2020;12(1):76.

18. Sankar P, Ahmed WN, Mariam Koshy V, Jacob R, Sasidharan S. Effects of COVID-19 lockdown on type 2 diabetes, lifestyle and psychosocial health: a hospital-based cross-sectional survey from South India. Diabetes Metab Syndr Clin Res Rev. 2020;14(6):1815-1819.

\section{SUPPORTING INFORMATION}

Additional supporting information may be found online in the Supporting Information section at the end of this article.

How to cite this article: D'Onofrio L, Pieralice S, Maddaloni E, et al. Effects of the COVID-19 lockdown on glycaemic control in subjects with type 2 diabetes: the glycalock study. Diabetes Obes Metab. 2021;1-7. https://doi.org/10.1111/dom.14380 